\title{
FDG PET/CT leads to the detection of metastatic involvement of the heart in non-Hodgkin's lymphoma
}

\author{
A. Julian • T. Wagner $\cdot$ L. Ysebaert • V. Chabbert • \\ P. Payoux
}

Received: 27 December 2010 / Accepted: 31 January 2011 /Published online: 23 March 2011

(C) Springer-Verlag 2011

We describe two rare cardiac findings on baseline FDG PET in histologically proven aggressive large B-cell non-Hodgkin's lymphoma (NHL).

The first case is a 21-year-old woman with stage IV NHL with a superior mediastinal mass and two lung nodules on contrast-enhanced CT images. FDG PET/CT showed intense uptake in these lesions and, without contiguity, a round pathological cardiac uptake in the apical septum and the right ventricle (a, b FDG PET images; $\mathbf{c}, \mathbf{d}$ fusion FDG PET/CT images; transverse and sagittal, respectively). The electrocardiogram showed negative T-waves in the septal location with left chest pain and dyspnoea. Transthoracic echocardiography confirmed the presence of a $3-\mathrm{cm}$ mass near the apex with development towards the right and left ventricles, without thrombus or pericardial effusion. Cardiac MR imaging revealed a hyperintense aspect on $\mathrm{T} 2$-weighted sequences, isointense on T1 sequences (e cine sequence T2-/T1-weighted image; $\mathbf{f}$ short-axis T2-weigted black blood SPIR image). This mass had disappeared on FDG PET/CT and echocardiography performed after two and four cycles of chemotherapy.

The second case is a 51-year-old man with stage III NHL on $\mathrm{CT}$ imaging who underwent FDG PET/CT revealing posterior parietal right auricular involvement (g transverse FDG PET image; $\mathbf{h}$ fusion FDG PET/CT image), confirmed on MR

A. Julian $(\bowtie) \cdot$ T. Wagner $\cdot$ P. Payoux

Department of Nuclear Medicine, CHU Purpan,

Place du Dr Baylac,

31059 Toulouse, France

e-mail: julian.a@chu-toulouse.fr

L. Ysebaert

Department of Haematology, CHU Purpan,

Toulouse, France

V. Chabbert

Department of Radiology, CHU Rangueil,

Toulouse, France imaging (i T1-weighted SE image, black blood, four chambers view). The FDG PET/CT scan at the end of treatment was normal.
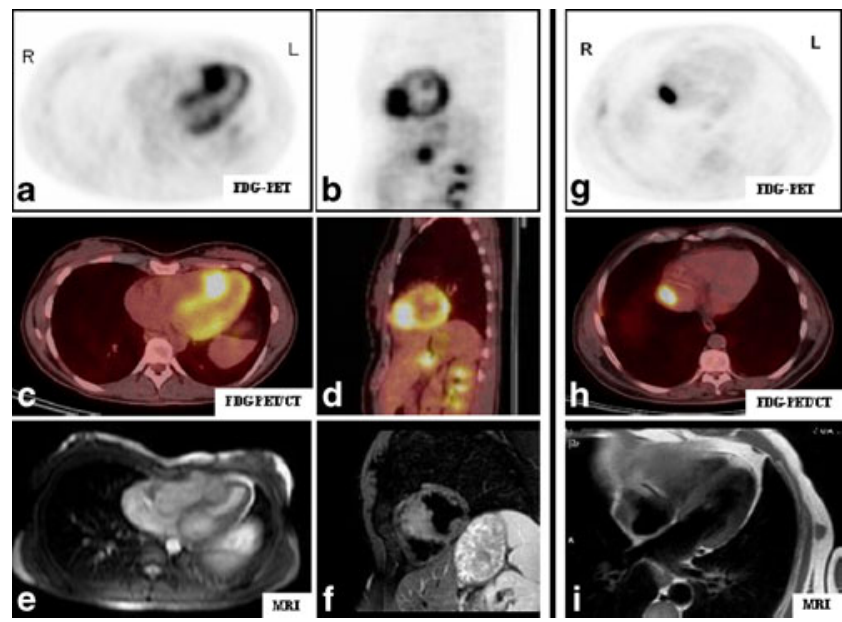

Metastases to the heart and pericardium are rare [1] in NHL but are associated with a poor prognosis, and are usually revealed by cardiac complications [2]. In these cases, FDG PET allowed early diagnosis and earlier chemotherapy, leading to the disappearance of cardiac locations before the occurrence of any cardiac complications, and therefore improving survival.

Conflicts of interest None.

\section{References}

1. Chiles C, Woodard PK, Gutierrez FR, Link KM. Metastatic involvement of the heart and pericardium: $\mathrm{CT}$ and MR imaging. Radiographics. 2001;21:439-49.

2. Van den Bos E, Van Gelder W, Baks T, Levin MD, Bakker J. Severe right ventricular inflow obstruction by non-Hodgkin lymphoma. Br J Haematol. 2008;142:858. 